\title{
Antigenic Community between Schistosoma mansoni and Biomphalaria glabrata: on the Search of Candidate Antigens for Vaccines
}

\author{
N Chacón, S Losada, B Noya, B Alarcón de Noya, O Noya ${ }^{+}$
}

Cátedra de Parasitología, Escuela "Luis Razetti” and Sección de Biohelmintiasis, Instituto de Medicina Tropical, Facultad de
Medicina, Universidad Central de Venezuela, Apartado Postal N 47.706, Los Chaguaramos 1041-A, Caracas, Venezuela

We have previously confirmed the presence of common antigens between Schistosoma mansoni and its vector, Biomphalaria glabrata. Cross-reactive antigens may be important as possible candidates for vaccine and diagnosis of schistosomiasis. Sera from outbred mice immunized with a soluble Biomphalaria glabrata antigen $(S \mathrm{Bg} A)$ of noninfected $\mathrm{B}$. glabrata snails recognized molecules of $\mathrm{SBg} A$ itself and $\mathrm{S}$. mansoni AWA by Western blot. Recognition of several molecules of the $S \mathrm{Bg} A$ were inhibited by pre-incubation with $A W A(16,30,36,60$ and $155 \mathrm{kDa})$. The only specific molecule of $A W A$, inhibited by $S \mathrm{Bg} A$, was a $120 \mathrm{kDa}$ protein. In order to determine which epitopes of $S \mathrm{Bg} A$ were glycoproteins, the antigen was treated with sodium metaperiodate and compared with non-treated antigen. Molecules of 140, 60 and $24 \mathrm{kDa}$ in the $\mathrm{SBg} A$ appear to be glycoproteins. Possible protective effects of the $\mathrm{SBg} A$ were evaluated immunizing outbred mice in two different experiments using Freund's Adjuvant. In the first one (12 mice/group), we obtained a significant level of protection (46\%) in the total worm load, with a high variability in worm recovery. In the second experiment (22 mice/group), no significant protection was observed, neither in worm load nor in egg production per female. Our results suggest that SBgA constitutes a rich source of candidate antigens for diagnosis and prophylactic studies.

Key words: cross reactions - antigens - Biomphalaria - Schistosoma - vaccine

Common antigens between different species of Schistosoma and their intermediate hosts have been reported (Dissous et al. 1986, Iwanaga 1994, Weston et al. 1994, Gamal-Eddin et al. 1996, 1997, Chacón et al. 2000). We demonstrated that sera from schistosome-infected persons reacted against soluble crude Biomphalaria glabrata antigen (SBgA) by ELISA (100\% of sensitivity) (Alarcón de Noya et al. 1989) and that sera from mice immunized with $\mathrm{S} B g \mathrm{~A}$ recognized several homologous snail molecules by Western-blot (Chacón et al. 2000).

Cross-reactive antigens may probably result from the adaptation of parasites to their invertebrate and vertebrate hosts and in consequence, could prevent immune recognition in the latter. Our basic hypothesis is that the parasite acquires snail molecules on its surface that would cover critical antigens, allowing its survival during skin penetration and the evasion from the protective immune mechanisms of definitive host during the first events of invasion.

This work has been financed by the Fonacit of the Minister of Science and Technology of Venezuela (Project no. S12000000564). Additional support was obtained by Bayer S.A. de Venezuela and the Coordinación de Investigación de la Facultad de Medicina de la Universidad Central de Venezuela for sponsoring the presentation of this paper in an international meeting.

${ }^{+}$Corresponding author. Fax: +58-212-605.3563 and -6930454. E-mail:noyaoo@yahoo.com/natychacon@yahoo.com

Received 18 June 2002

Accepted 15 August 2002
In the present work, non-singenic mice were immunized with a crude antigen of non-infected B. glabrata in order to demonstrate, first, the cross-reactivity and crossinhibition between $S$. mansoni and B. glabrata common proteins. Second, to characterize $\mathrm{S} B g \mathrm{~A}$, particularly its glycoprotein components and third, to determine the protective effect of the crude preparation of B. glabrata in mice against $S$. mansoni infection.

\section{MATERIALS AND METHODS}

Crude $\mathrm{SBg} A$ - The $\mathrm{S} B g \mathrm{~A}$ was obtained from the body of non-infected snails manually homogenized with PBS Tween-20 $0.05 \%$ during $30 \mathrm{~min}$ in ice bath. The homogenate was centrifuged at $2,000 \mathrm{~g}$ for $20 \mathrm{~min}$ at $4^{\circ} \mathrm{C}$ and the recovered supernatant was used as $\mathrm{S} B g \mathrm{~A}$. Protein was quantified by Bradford (1976).

Adult worm antigen (AWA) - Adult S. mansoni worms JL strain (kindly provided by Dr Italo Cesari, Instituto Venezolano de Investigaciones Científicas) were collected from hamsters six to seven weeks after experimental infection, using the perfusion method of Smithers and Terry (1965). AWA was prepared as described by Noya et al. (1995). Worms were washed three times in cold saline solution $(0.85 \% \mathrm{NaCl})$, homogenized in phosphate-buffered saline (PBS) containing protease inhibitors $(1 \mathrm{mM}$ PMSF and $1 \mathrm{mM}$ EDTA) in an ice bath and centrifuged at $12,000 \mathrm{~g}$ for $2 \mathrm{~h}$ at $4^{\circ} \mathrm{C}$. The supernatant containing AWA was recovered and stored in small aliquots at $-80^{\circ} \mathrm{C}$ until used. Protein concentrations were determined as described by Bradford (1976).

Cross-inhibition of the recognition of $\mathrm{SBg} A$ molecules by SDS-PAGE with AWA - The SBgA antigen was separated by SDS-PAGE under reducing and dissociating 
conditions (Laemmli 1970) in 12.5\% mini-gels and transferred onto nitrocellulose membrane. Thereafter, the strips were exposed to mice immune sera diluted 1:100 in 5\% non-fat milk in PBS with $0.05 \%$ Tween 20 (blocking solution), incubated for $60 \mathrm{~min}$ with anti-mouse IgG biotin conjugate (Sigma) diluted 1:20000 and then incubated for $30 \mathrm{~min}$ with avidin-peroxidase (Sigma) diluted 1:30000. Each step was followed by three washes, respectively.

The two first nitrocellulose strips were used to test the recognition of the original molecules of $\mathrm{S} B g \mathrm{~A}$ by nonimmune sera and immune sera. Each mouse immune serum was pre-incubated for $30 \mathrm{~min}$, with $\mathrm{SBgA}$ or AWA before the exposure to the strip of nitrocellulose. If the immune serum contains antibodies against the antigen, they will be depleted during pre-incubation and will not be available to $\mathrm{SBgA}$ in the electrotransferred membrane immunoblot, inhibiting the recognition of the common molecules between $S$. mansoni and B. glabrata. Finally, strips were washed and incubated with a chemiluminiscent substrate for detection of peroxidase (HRP) (Super Signal ${ }^{\circledR}$ West Pico, Pierce) and exposed to a film (Hyperfilm ${ }^{\mathrm{TM}} \mathrm{ECL}^{\mathrm{TM}}$ Amersham Pharmacia Biotech).

Cross-inhibition of the recognition of AWA molecules by $S D S-P A G E$ with $S \mathrm{Bg} A$ - Also AWA was separated by SDS-PAGE in $10 \%$ mini-gels and transferred onto nitrocellulose membrane. As previously described for the cross-inhibition of the recognition of $\mathrm{SBgA}$ molecules, we used anti-mouse $\operatorname{IgG}$ biotin conjugate and then incubated with avidin-peroxidase. The two first nitrocellulose strips were used to test the recognition of the original molecules of AWA by non-immune sera and immune sera, respectively. Additionally, each mouse immune serum was pre-incubated for $30 \mathrm{~min}$, with $\mathrm{S} B g \mathrm{~A}$ or AWA before the nitrocellulose strip exposure. If the immune sera contained antibodies against the pre-incubated antigen, it will not have antibodies available to react against AWA in the immunoblot, therefore the recognition of the common molecules between B. glabrata and S. mansoni will be inhibited.

$S \mathrm{Bg} A$ glycoproteins composition - After the electrophoresis and transfer of $\mathrm{S} B g$ A to nitrocellulose membrane, the strips were incubated in different concentrations of sodium metaperiodate (SMP) to determine the optimal concentration of SMP. Following the method of Woodward et al. (1985) modified by Alarcón de Noya et al. (2000), we incubated the blocked nitrocellulose strips in $10 \mathrm{mM}$ SMP. After this treatment, tested sera were diluted 1:100 in blocking solution. The anti-mouse IgG biotin conjugated plus avidin-peroxidase was used. Finally, strips were washed and incubated $2 \mathrm{~min}$ in an enhaced chemiluminescent substrate for detection of peroxidase (HRP) and exposed to a high performance chemiluminescence film as described above.

Protection assays - Two separate experimental protection assays were carried out using non-singenic white mice. Before the first immunization, we obtained blood samples (non-immune sera) from mice divided in one experimental group and one control group. The experimental group was subcutaneously immunized with $200 \mu \mathrm{g}$ of SBgA plus Complete Freund's Adjuvant (CFA) (1:1 v/v) in the first dose and Incomplete Freund's Adjuvant (IFA) in the two successive doses at two weeks interval. The control group was immunized only with CFA/IFA. Two weeks after the last immunization another blood sample was obtained (immune sera) and mice were challenged with 100 cercariae by the tail immersion method (Cesari \& Alarcón de Noya 1987, Chacón 2000). Eight weeks after infection, mice were sacrificed and parasites were recovered by perfusion of the hepatic portal system (Smithers $\&$ Terry 1965).

Protection level of the $\mathrm{S} B g$ A was estimated according to the formula by Smithers et. al. (1989):

$$
1-\left[\frac{\mathrm{B}}{\mathrm{A}}\right] \times 100
$$

A : mean worm load of the group of mice immunized with adjuvant (control group); and

B: mean worm load of the group of mice immunized with $\mathrm{S} B g$ A (experimental group).

Other parameters like the maturity of worms (Chacón 2000), sex, feces egg count by Kato-Katz (Katz et al. 1972) and viable eggs in hepatic tissue (Cheever 1968) were performed only in the second experiment. In the first experiment, 12 mice per group were used, while 22 mice per group were used for the second experiment.

Statistical analysis was carried out using the Student's t-test.

\section{RESULTS}

Cross-inhibition of the recognition of $\mathrm{SBg} A$ molecules by SDS-PAGE with AWA - Immune sera recognized in $S B g A$ 's immunoblot nine specific molecules: 155,122 , $97,90,60,50,36,31$ and $25 \mathrm{kDa}$ (Fig. 1). All these molecules were completely inhibited by the pre-incubation with $\mathrm{S} B g \mathrm{~A}$ antigen in different frequencies that ranged from $10 \%$ to $60 \%$ of all mice sera studied. Only five molecules were inhibited by AWA $(155,60,36,31$ and $25 \mathrm{kDa})$, but with lower frequencies. The molecule of $60 \mathrm{kDa}$ was inhibited by $18 \%$ sera of mice.

Cross-inhibition of the recognition of AWA molecules by SDS-PAGE with $S \mathrm{Bg} A$ - Immune sera recognized only one specific molecule of $120 \mathrm{kDa}$ in AWA (Fig. 2). This molecule was completely inhibited by AWA by the $63 \%$ mice sera. Also, it was cross-inhibited by the $56 \%$ mice sera when pre-incubated with $\mathrm{S} B g \mathrm{~A}$.

$S B g A$ glycoproteins composition - The treatment of SBgA with SMP revealed four possible glycosilated epitopes: 130, 60, 50 and $25 \mathrm{kDa}$ (Fig. 3).

Protection assay - In the first experiment, using 12 mice per group, $46 \%$ of protection was demonstrated, based on the reduction of adult worms. But, when we duplicated the experiment with 22 mice per group, there was not any protective effect against $S$. mansoni infection (Fig. 4), even when a significant increase in immature worms was observed.

All the protection parameters studied in the second experiment are shown in the Table.

\section{DISCUSSION}

Over the past two decades, several investigators have demonstrated the antigenic community between $S$. mansoni and Biomphalaria (Dissous et al. 1986, 1990, Iwanaga et al. 1992, Iwanaga 1994, Weston et al. 1994). 
The possible role of these shared antigens in immune evasion in the host or in the maintenance of antigenic parasite polymorphism has to be elucidated. It has been suggested that at least some of these shared antigens reflect a genetic accommodation between the host and the parasite ("molecular mimicry") as a result of the pressure of selection (Damian 1987).
Our cross-inhibition or immunoadsorption experiments further demonstrate the antigenic community between S. mansoni and one of its vectors, B. glabrata. Indeed, as it was expected, several molecules were inhibited and the frequencies of inhibition were higher when the immune sera (containing anti-snail antibodies) were pre-incubated with the autologous antigen. But the incu-

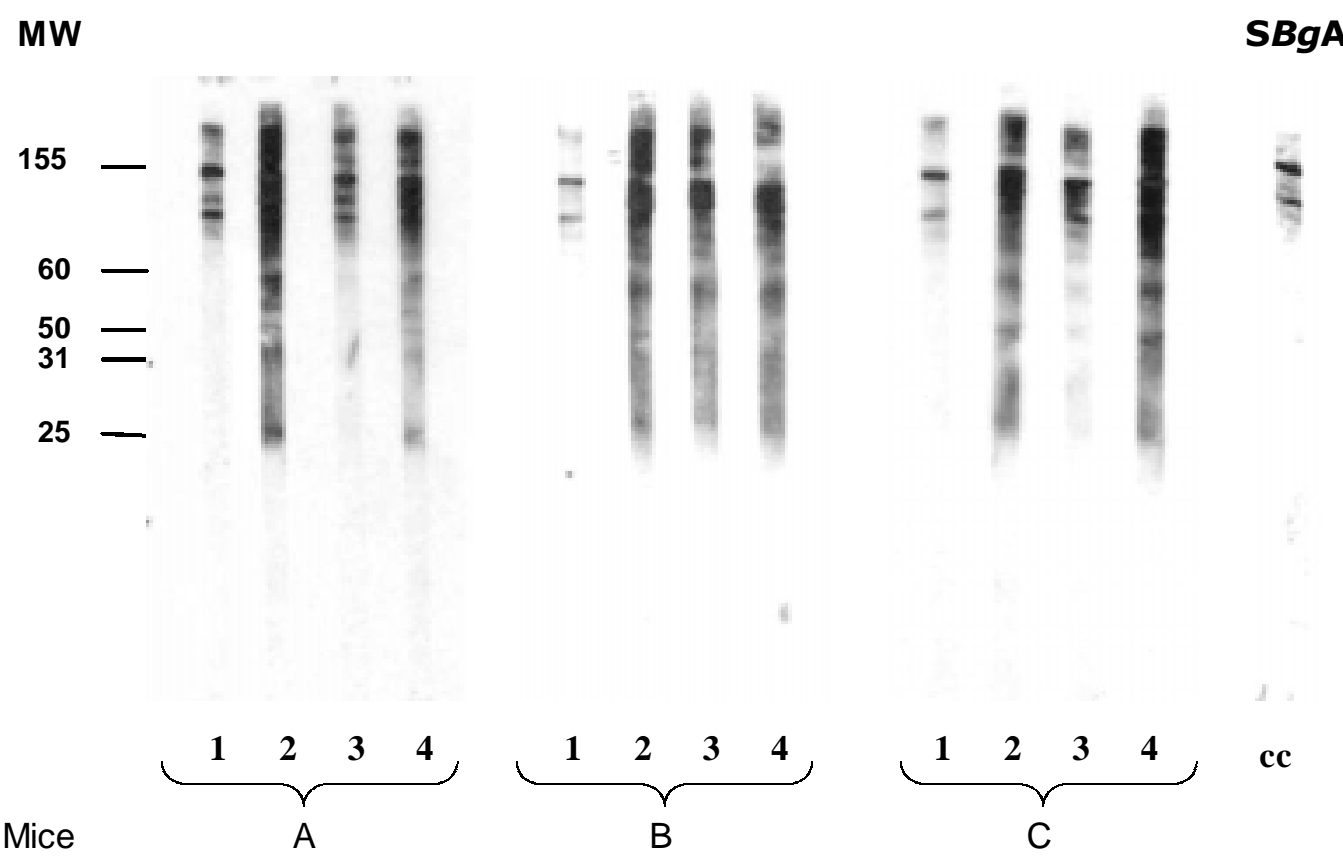

Fig. 1: pattern of recognition by Western-Blot $(\alpha-\mathrm{S} B g \mathrm{~A})$ of soluble Biomphalaria glabrata antigen ( $\mathrm{S} B g \mathrm{~A})$ of three representative mice immunized with the homologous antigen and preincubated with Schistosoma mansoni adult worm antigen (AWA) and SBgA. 1. preimmune sera; 2. immune sera; 3. pre-incubation of immune sera with SBgA; 4. preincubation of immune sera with AWA; cc: conjugate control.

MW

AWA
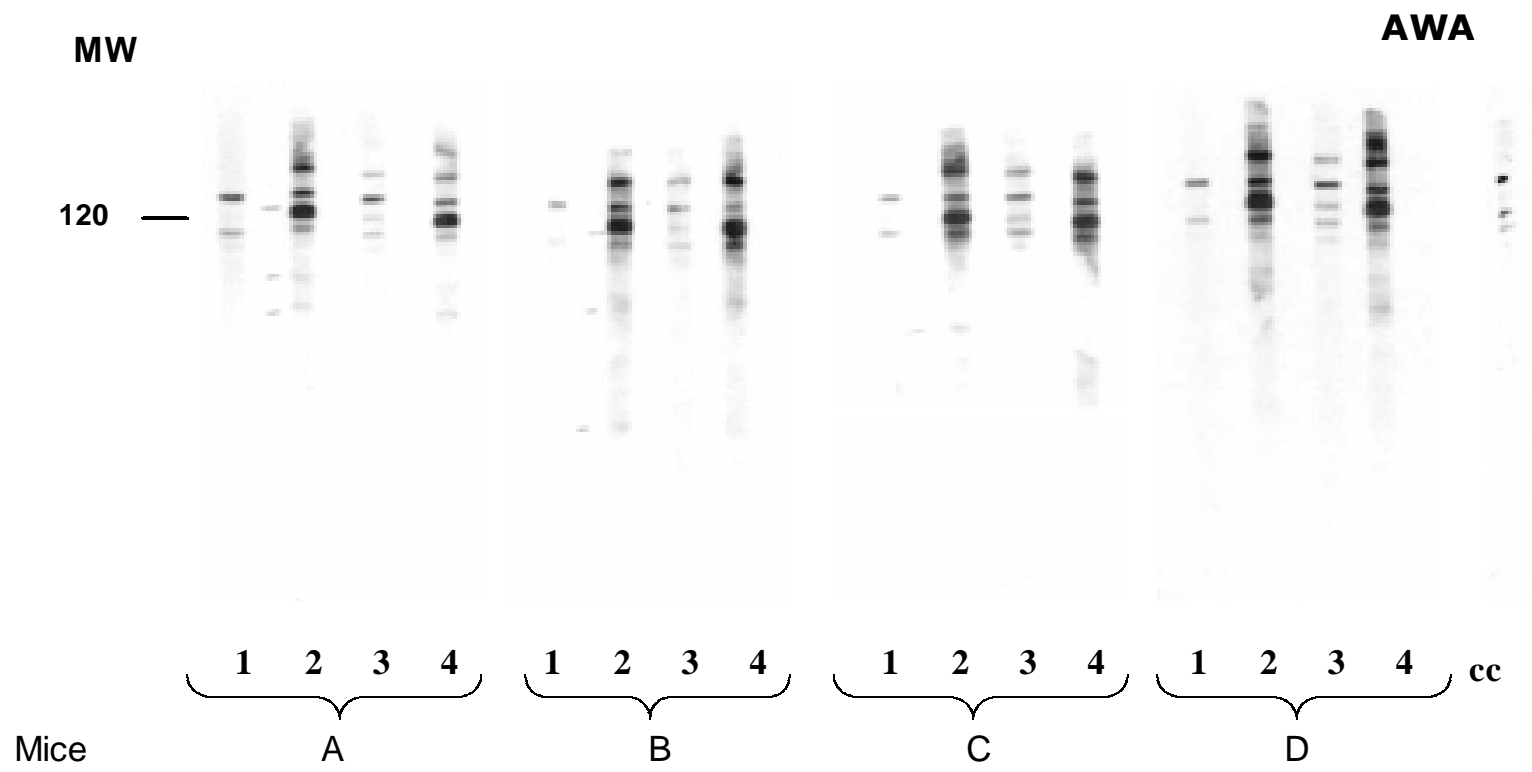

Fig 2: pattern of recognition of adult worm antigen (AWA) by four representative mice immunized with soluble Biomphalaria glabrata antigen ( $\mathrm{SBgA}$ ) and preincubated with $\mathrm{SBgA}$ and Schistosoma mansoni AWA, by Western-Blot. 1. pre-immune sera; 2 . immune sera; 3. pre-incubation of immune sera with $\mathrm{S} B g \mathrm{~A}$; 4. pre-incubation of immune sera with AWA; cc: conjugate control. 


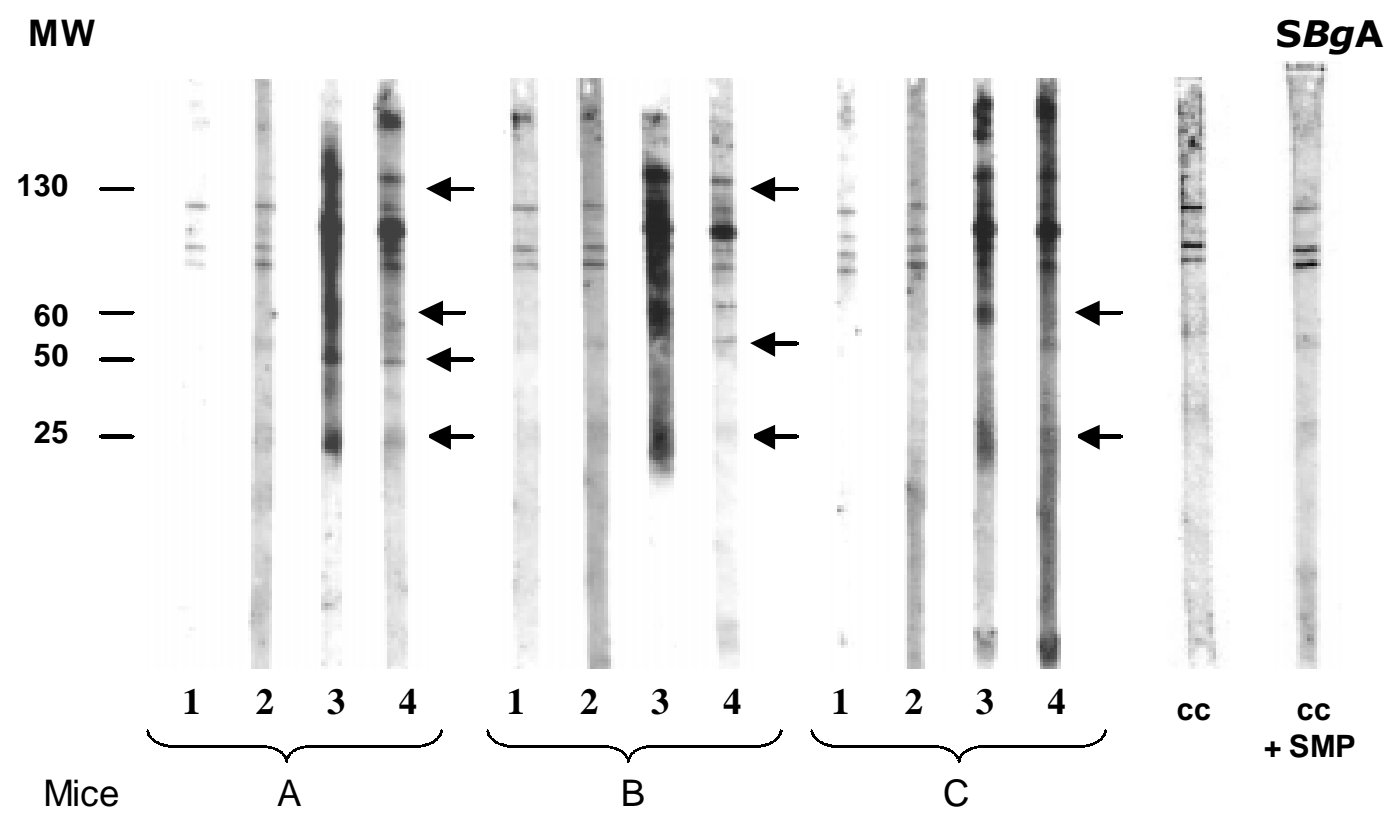

Fig 3: recognition of possible Biomphalaria glabrata glycoproteins by immunoblot after treatment with sodium metaperiodate (SMP). 1 pre-immune sera; 2. soluble Biomphalaria glabrata antigen (SBgA) treated with sodium metaperiodate (SMP) and incubated with preimmune sera; 3 . immune sera; 4 . SBgA treated with SMP and incubated with immune sera; cc: conjugate control; cc + SMP: conjugate control treated with SMP.

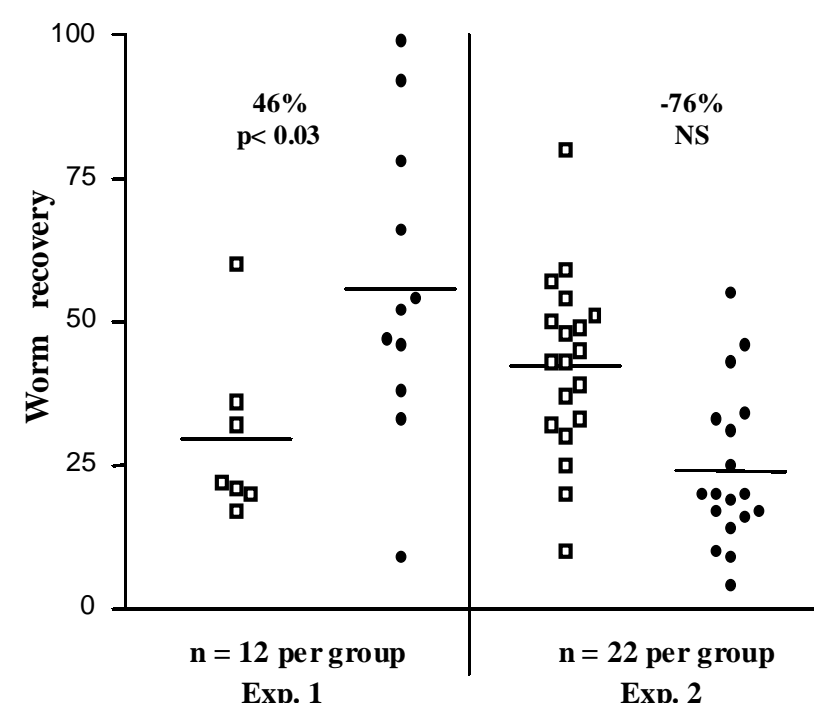

Fig 4: protection of outbred mice against infection of Schistosoma mansoni by vaccination with soluble Biomphalaria glabrata antigen $(\mathrm{S} B g \mathrm{~A})$; $\mathbf{\square}$ Vaccinated group; $\bullet$ Control group (Freund's Adjuvant only).

bation with heterologous antigen also inhibited the recognition of certain molecules, reinforcing the hypothesis of antigenic community between the parasite and its intermediate host.

Different molecules were inhibited in each case at different frequencies, showing a variable response from one animal to another, indicating the complexity of the im-
TABLE

Parameters evaluated in the second experiment of protection against infection with Schistosoma mansoni using outbred mice immunized with soluble Biomphalaria glabrata antigen

\begin{tabular}{lcc}
\hline Parameters & $\begin{array}{c}\text { Vaccinated group } \\
\mathrm{X} \pm \mathrm{SD}(\mathrm{n}=22)\end{array}$ & $\begin{array}{c}\text { Control group } \\
\mathrm{X} \pm \mathrm{SD}(\mathrm{n}=22)\end{array}$ \\
\hline Total number of worms & $42.37 \pm 3.62$ & $24.06 \pm 3.23$ \\
No. females & $16.84 \pm 1.91$ & $9.88 \pm 1.38$ \\
No. males & $25.47 \pm 2.13$ & $14.72 \pm 2.12$ \\
In copula & $5.57 \pm 0.87$ & $3.94 \pm 0.92$ \\
Mature worms & $40.26 \pm 3.60$ & $23.3 \pm 3.14$ \\
Immature worms & $2.10 \pm 0.47 a$ & $0.72 \pm 0.22$ \\
No. eggs/g of feces & $583.9 \pm 78.63$ & $309.9 \pm 36.02$ \\
No. eggs/g of liver & $0.014 \pm 0.003$ & $0.005 \pm 0.007$ \\
No. eggs/g feces/female & $39.66 \pm 18.44$ & $48.82 \pm 67.15$ \\
\hline
\end{tabular}

$a: \mathrm{p}<0.01$

mune response. When exposed to the autologous antigen $(\mathrm{SBgA}), 60 \%$ of mice sera did not recognize the 97 $\mathrm{kDa}$ and $60 \mathrm{kDa}$ molecules, and only the latter molecule was cross-inhibited by AWA. On the other hand, the only specific molecule of AWA was a $120 \mathrm{kDa}$ protein, which was inhibited in a similar high frequency by $\mathrm{SBgA}(56 \%$, heterologous antigen) and by AWA (63\%). Norden et al. (1982) purified a $120 \mathrm{kDa}$ protein from cercariae and a 170 $\mathrm{kDa}$ protein from male and female worms using a monoclonal antibody and discussed the relevance of this antibody for serodiagnosis and immunoprophylaxis. It will be interesting to confirm if it is the same antigen described by Norden et al. (1982) present in different stages of the 
parasite (cercariae, schistosomula, adult, egg and miracidia). Apparently, this protein contains common epitopes between the two organisms under study.

Evidences were given to support the importance of carbohydrate epitopes in antigenic mimicry between $S$. mansoni and its intermediate host B. glabrata (Dissous et al. 1986, 1990). It was demonstrated that four possible glycosilated epitopes after SMP treatment of $\mathrm{S} B g \mathrm{~A}$ might be related with the cross-reactivity observed between the parasite and the mollusk, as described by Dissous et al. (1986). The role of cross-reactive glycosilated and not glycosilated antigens in host-parasite adaptation needs to be investigated either for diagnostic and/or prophylaxis in several Schistosoma experimental models and their corresponding intermediate hosts.

Our basic hypothesis is that the parasite could acquire snail molecules on its surface that would cover critical antigens during the skin penetration of the definitive host (Devine \& Kemp 1984), or alternatively, share epitopes with its intermediate host (B. glabrata). Therefore, if immunized animals with the whole crude snail antigen are able to induce anti-snail antibodies against epitopes present in the newly transformed schistosomula, it would be possible to elicit protective anti-schistosomula antibodies.

In our first experiment, $46 \%$ of protection was demonstrated based only on worm burden. So, the protective potential of the non-infected crude B. glabrata antigen was repeated in a second experiment, not only in terms of worm load but also in terms of maturity of worms, feces egg count by Kato-Katz and viable eggs in hepatic tissue. The number of animals per group of study was also incremented in this experiment. No significant differences could be demonstrated for total worm load, sex, worm maturity, number of worms in copula, number of eggs/g of feces, number of eggs/g of liver and number of eggs/ $\mathrm{g}$ of feces/female. The only significant difference was seen over the mean of immature worms. It is important to mention that the control group that received the Freund's adjuvant in this study, showed a reduced worm load and anti-fecundity effect described by others (Wasilewski et al. 1996, Chacón 2000). In this study, while the whole crude snail antigen did not protect immunized mice, the use of a purified antigen might eventually show protection. In fact, Gamal-Eddin et. al. (1996) reported a reduction in worm load (36-44\%) using two purified non-infected hepatopancreas of $B$. alexandrina antigens. They also reported a reduction in the viable eggs in tissue and the number of worms in copula.

In order to clearly demonstrate the protective effect of any antigen, we suggest to use a significant number of animals and at least triplicate experiments.

The snail, B. glabrata constitutes a rich source of antigen for serodiagnostic and prophylactic studies for $S$. mansoni. The $\mathrm{S} B g$ A is easy to obtain, with a high recovery of proteins and low cost, compared to other parasite antigens, like AWA and SEA.

Further experiments are required in order to better characterize the cross-reactive and non cross reactive snail antigens that are preferentially recognized by resistant experimental animals and humans.

\section{ACKNOWLEDGEMENTS}

To Dr Jaime Torres for his assistance in reviewing this manuscript.

\section{REFERENCES}

Alarcón de Noya B, Colmenares C, Lanz H, Caracciolo, MA, Losada S, Noya O 2000. Schistosoma mansoni: immunodiagnosis is improved by sodium metaperiodate which reduces cross-reactivity due to glycosilated epitopes of soluble egg antigen. Exp Parasitol 95: 106-112.

Alarcón de Noya B, Spencer L, Noya O 1989. Comunidad antigénica de Schistosoma mansoni y Biomphalaria glabrata: su evaluación seroepidemiológica. Act Cient Venez 40 (Supl. 1): 147.

Bradford MM 1976. A rapid and sensitive method for the quantitation of microgram quantities of protein utilizing the principle of protein-dye binding. Anal Biochem 72: 248 .

Cesari IM, Alarcón de Noya B 1987. Esquistosomiasis Mansoni: Diagnóstico y Control. Manual de Campo y de Laboratorio, Instituto Venezolano de Investigaciones Científicas, Caracas, $120 \mathrm{pp}$.

Chacón N 2000 Inmunoprofilaxis con Péptidos Sintéticos Derivados de Antígenos Enzimáticos de Schistosoma mansoni en el Modelo Múrido, Instituto Venezolano de Investigaciones Científica, Caracas, $170 \mathrm{pp}$.

Chacón N, Losada S, Noya B, Alarcón de Noya B, Noya O 2000. The protective effect of crude antigens of Biomphalaria glabrata against Schistosoma mansoni. XVth International Congress for Tropical Medicine and Malaria, Cartagena, Colombia, 2: 94.

Cheever AW 1968. Conditions affecting the accuracy of potassium hydroxide digestion techniques of counting Schistosoma mansoni eggs in tissues. Bull WHO 39: 328-331.

Damian RT 1987. Molecular mimicry. Parasitol Today 3: 263 266.

Devine DV, Kemp WM 1984. Immunocytochemical location of molluscan host-like antigenic determinants on the surfaces of adult Schistosoma mansoni. J Parasitol 70: 172-174.

Dissous C, Grzych JM, Capron A 1986. Schistosoma mansoni shares a protective oligosaccharide epitope with freshwater and marine snails. Nature 323: 443-445.

Dissous C, Torpier G, Duvaux-Miret O, Capron A 1990. Structural homology of tropomyosins from the human trematodes Schistosoma mansoni and its intermediate host Biomphalaria glabrata. Mol Biochem Parasitol 43:245256.

Gamal-Eddin FM, Fayed MA, Imam MH, Bayoumi AMS, Elkady MA, Abdel-Raheem MA 1996. The immunogenic effect of purified antigens of Biomphalaria alexandrina against Schistosoma mansoni in experimental animals (I) as measured by worm load and viability of ova. J Egypt Soc Parasitol 26: 609-617.

Gamal-Eddin FM, Fayed MA, Imam MH, Bayoumi AMS, Shahat A, Abdel-Raheem MA, El-Sayed GA, El-Kady MA, Ghanam ME 1997. The immunogenic effect of purified antigens of Biomphalaria alexandrina against Schistosoma mansoni in experimental animals (II) as measured by the histopathological changes in the liver. J Egypt Soc Parasitol 27: 153-156.

Iwanaga Y 1994. Studies on host-parasite relationship between the Puerto Rican strain of Schistosoma mansoni and Biomphalaria snails. Southeast Asian J Trop Med Public Health 25: 509-515.

Iwanaga Y, Valfrido de Santana J, Goncalves JF 1992. Studies on common antigenicities between the Belo Horizonte strain (Brazil) of Schistosoma mansoni eggs and Biomphalaria 
snails by inmunoelectrophoresis. Southeast Asian J Trop Med Public Health 23: 98-102.

Katz N, Chavez A, Pellegrino J 1972. A simple device for quantitative stool thick smear technique in schistosomiasis mansoni. Rev Inst Med Trop São Paulo 14: 397-400.

Laemmli UK 1970. Cleavage of structural proteins during the assembly of the head of bacteriophage T4. Nature 227: 680-685.

Norden AM, Aronstein WS, Strand M 1982. Schistosoma mansoni: identification, characterization and purification of the spine glycoprotein by monoclonal antibody. Exp Parasitol 54: 432-442

Noya O, Fermín Z, Alarcón de Noya B, Losada S, Colmenares C, Hermoso T 1995. Humoral immune response of children with chronic schistosomiasis. Isotype recognition of adult worm antigens. Paras Immunol 17: 319-328.

Smithers SR, Terry RJ 1965 . The infection of laboratory hosts with cercariae of Schistosoma mansoni and the recovery of adult worm. Parasitology 55: 695-700.

Smithers SR, Hackett F, Ali PO, Simpson AJG 1989. Protective immunization of mice against Schistosoma mansoni with purified adult worm surface membrane. Paras Immunol 11: 301-318.

Wasilewski MM, Lim KC, Philips J, McKerrow JM 1996. Cysteine protease inhibitors block schistosome haemoglobin degradation in vitro and decrease worm burdens and egg production in vivo. Mol Biochem Parasitol 81: 179-189.

Weston D, Allen B, Thakur A, LoVerde PL, and Kemp WM 1994. Invertebrate host-parasite relationship: convergent evolution of a tropomyosin epitope between Schistosoma sp., Fasciola hepatica and certain pulmonate snails. Exp Parasitol 78: 269-278.

Woodward M, Young W, Bloodgood RA 1985. Detection of monoclonal antibodies specific for carbohydrate epitopes using periodate oxidation. J Immunol Meth 78: 143-153. 